\title{
Growth of textured films of BiSCCO from alkali halide solutions
}

\author{
KATTA NARASIMHA REDDY*, U V SUBBA RAO, T S P L N \\ PRASAD and E LAXMI NARSAIAH \\ Department of Physics, Osmania University, Hyderabad 500007, India. \\ *Also, Istituto di Electtronica dello Stato Solido del C. N. R., via Cineto Romano 42 I-00156 \\ Roma, Italy.
}

\begin{abstract}
Films of $\mathrm{Bi}-\mathrm{Sr}-\mathrm{Ca}-\mathrm{Cu}-\mathrm{O}$ with lead doping using $\mathrm{SrF}_{2}$ substrates have been grown from $\mathrm{KCl}$ solution. The films were highly textured with $C$-axis perpendicular to the substrate surface. The onset of the resistive transition was at $81 \mathrm{~K}$ and zero resistance at $78 \mathrm{~K}$. This technique is promising in view of the possibility to grow large films of both $\mathrm{Bi}_{2} \mathrm{Sr}_{2} \mathrm{CaCu}_{2} \mathrm{O}_{x}$ and $\mathrm{Bi}_{2} \mathrm{Sr}_{2} \mathrm{Ca}_{2} \mathrm{Cu}_{3} \mathrm{O}_{y}$ phases.
\end{abstract}

Keywords. Doping; resistive transition; zero resistance.

\section{Introduction}

Since the discovery of high temperature superconductors based on cuprates (Bednorz and Muller 1986) it appears probable that superconductors will find applications in the form of thin films in solid-state devices like superconducting quantum-interference devices and or particle detectors operating at liquid nitrogen temperatures. Another major area where low-loss properties of superconductors will have potential impact is in devices operating at microwave, radio and mm-wave frequencies. However, one major obstacle is the inability to deposit them as films on low-loss and low dielectric constant insulators which are also compatible with compound semiconductors. A class of materials that satisfy the above criteria are alkaline earthfluorides which have static dielectric constants between 6 and 8 and large band gaps of the order of $10 \mathrm{eV}$ at room temperature. These fluorides are also stable and have been demonstrated to be chemically compatible with compound semiconductors like GaAs and InP. Therefore $\mathrm{SrF}_{2}$ single crystals were used as substrates to grow films of BiSCCO. In the present paper, we report the growth of $\mathrm{Bi}-\mathrm{Sr}-\mathrm{Ca}-\mathrm{Cu}-\mathrm{O}$ films with lead-doping from $\mathrm{KCl}$ solutions onto (001)-oriented $\mathrm{SrF}_{2}$ substrates.

\section{Experimental}

The melt, whose composition is given in table 1 , was prepared as follows. The oxides and carbonates (all at least $99.5 \%$ pure) constituting BiSCCO were weighed in the atom ratios $\mathrm{Pb}: \mathrm{Bi}: \mathrm{Sr}: \mathrm{Ca}: \mathrm{Cu}=0.66: 1.33: 2: 1: 2$, thoroughly mixed in an agate mortar, and relatively a thick layer of this mixed powder was spread onto a number of substrates. These substrates were kept in a wide-mouthed platinum crucible so that no two substrates touch each other. The platinum crucible containing these substrates was filled with about $120 \mathrm{~g}$ of $\mathrm{KCl}$ powder and placed in a vertical tubular furnace, heated at about $800^{\circ} \mathrm{C}$ for $24 \mathrm{~h}$ and then switched off. The crucible was removed from the furnace and the substrates separated from the crucible by dissolving the residual-solidified melt in distilled water. The film thickness was measured by a mechanical profile meter. An average thickness of $2-3 \mu \mathrm{m}$ was measured. 


\section{Results and discussion}

X-ray diffraction spectra of the films were taken using a Bragg-Brentano diffractometer and $\mathrm{CoK}_{\alpha}$ radiation. A typical spectrum is shown in figure 1 . The film consisted of the $\mathrm{Bi}_{2} \mathrm{Sr}_{2} \mathrm{CaCu}_{2} \mathrm{O}_{8+x}$ phase. The diffraction peaks in the figure were indexed on the basis of the above structure. The film peaks have Miller's indexes of the (001) type indicating a strong preferential orientation of the film with the $C$-axis perpendicular to the substrate surface. The $C$-lattice parameter was measured using the $(0032)$ reflection. The value of $30.81 \AA$ is in good agreement with those reported for polycrystalline samples of the $\mathrm{Bi}_{2} \mathrm{Sr}_{2} \mathrm{CaCu}_{2} \mathrm{O}_{8-x}$ compound (Hazen 1988; Balestrino et al 1989) and indicates that no chemical impurities, either from the substrate or the solvent, were included in the film.

Table 1. Composition of the solution used for the growth of BiSCCO films

\begin{tabular}{lrcc}
\hline & Mole \% & Moles & Grams \\
\hline $\mathrm{PbO}$ & 0.86 & 0.0150 & 3.35 \\
$\mathrm{Bi}_{2} \mathrm{O}_{3}$ & 0.86 & 0.0150 & 6.99 \\
$\mathrm{SrCo}_{3}$ & 2.57 & 0.0450 & 6.65 \\
$\mathrm{CaCo}_{3}$ & 1.28 & 0.0225 & 2.28 \\
$\mathrm{CuO}$ & 2.57 & 0.0460 & 3.6 \\
$\mathrm{KCl}$ & 91.86 & 1.6090 & 120.0 \\
\hline
\end{tabular}

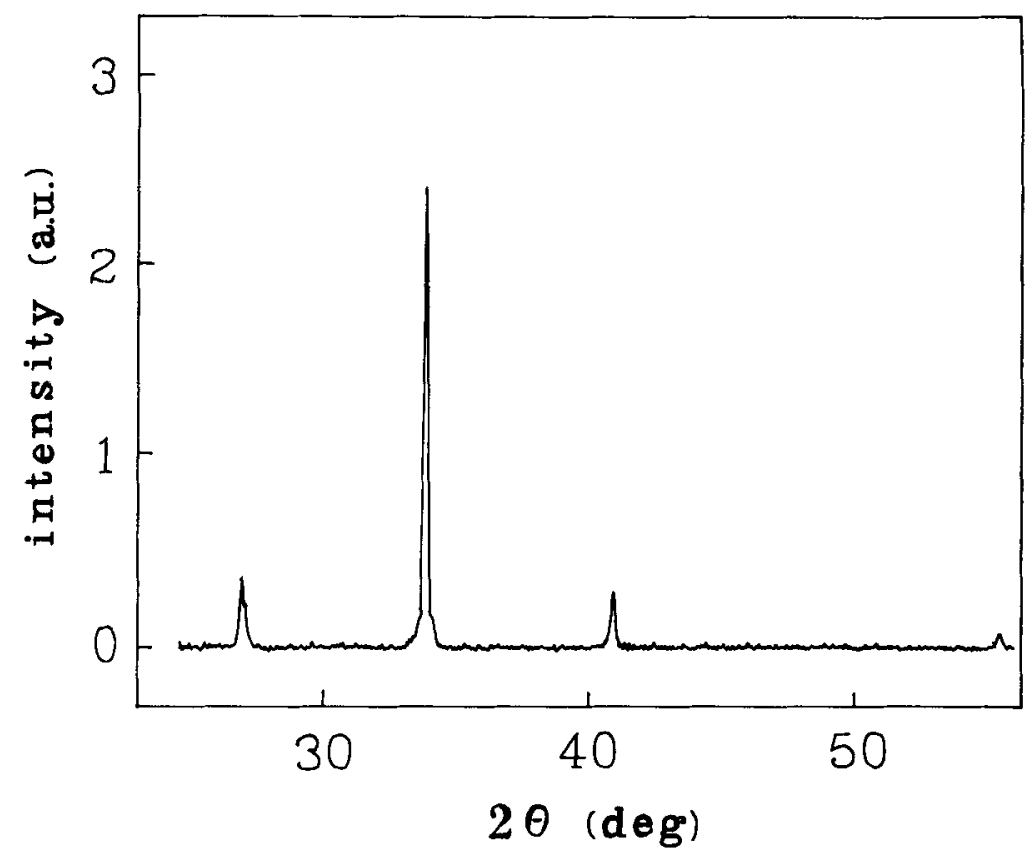

Figure 1. X-ray diffraction spectrum of a film $\mathrm{CoK}_{\alpha}$ radiation. 


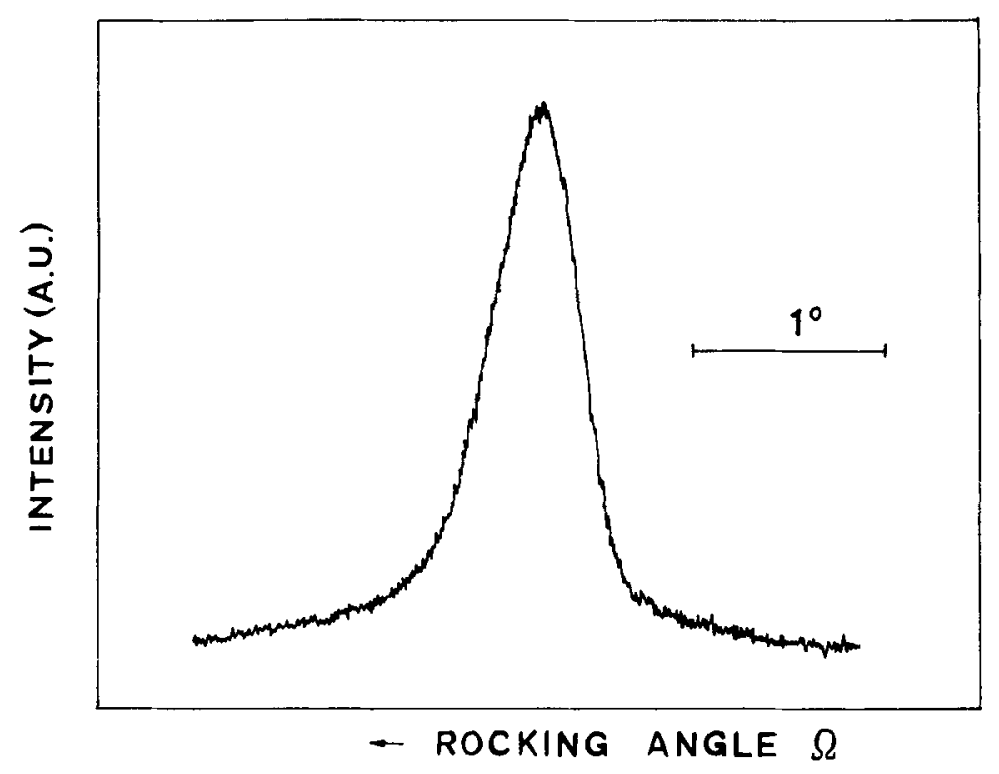

Figure 2. Rocking curve of the BiSCCO film (0012) reflection $\mathrm{CoK}_{\alpha}$ radiation.

The degree of texture of the film was measured by the $\theta$-scan technique using a Bragg-Brentano diffractometer. The detector was set at the angle of the most intense $(0012)$ reflection. While the sample was rotated around the goniometer axis, (again $\mathrm{CoK}_{\alpha}$ radiation was used) the incident beam was collimated by a $1 / 12^{\circ}$ divergence slit. The resulting rocking curve is shown in figure 2. The FWHM value of $0.5^{\circ}$ gives a good estimate of the mosaic spread along the $C$-axis.

To measure the resistance, four electrical contacts were obtained on the films by silver paint. The contact resistance was of the order of $\mu \Omega$. Figure 3 shows the temperature behaviour of the resistance $R$ obtained by measuring the voltage drop induced by injecting a dc current of $10 \mu \mathrm{A}$. It is seen that $R$ has a metallic behaviour decreasing linearly from room temperature down to the resistive transition. The extrapolated residual resistance at $0 \mathrm{~K}$ was about $1 / 10$ th of the resistance at room temperature. At $81 \mathrm{~K}$ the superconducting onset occurs and zero resistance reached at $78 \mathrm{~K}$. Several variations with respect to the above described growth procedures, concerning melt composition and preparation, gave no observable differences in the grown films.

\section{Conclusions}

Our results indicate that films grown using liquid solution are quite promising. This technique yields films of 2223 phase of BiSCCO and constitutes a major improvement in the development of this class of materials, since the $2223 \mathrm{BiSCCO}$ phase thus far has been obtained only as minority phase in the 2212 BiSCCO pellets and subjected to long treatments at temperatures very close to the melting point. This technique can lead to the growth of films of large size with high crystallographic quality useful for many fundamental investigations. 


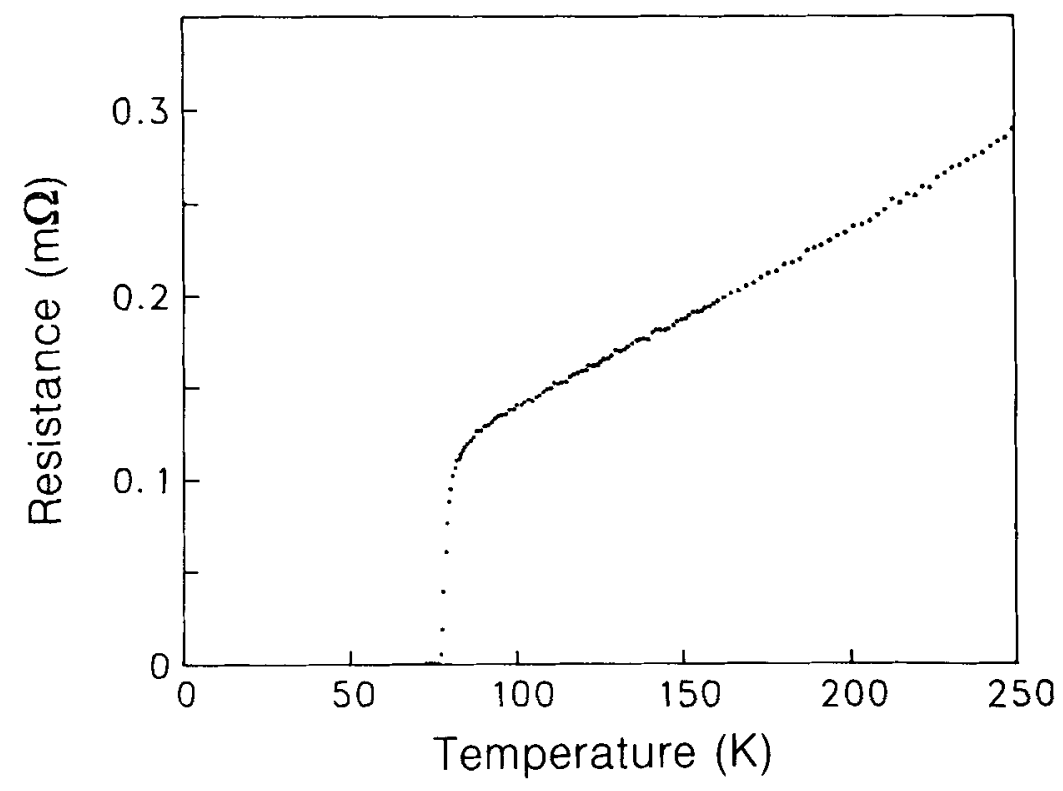

Figure 3. Resistance vs temperature for a BiSCCO film.

\section{Acknowledgements}

One of the authors (KNR) thanks the International Centre for Theoretical Physics for a research fellowship to work at IESS-CNR Roma. He also acknowledges helpful discussions and encouragement received from Prof. P Paroli of IESS-CNR Roma.

\section{References}

Balestrino $G_{4}$ Paoletti A. Paroli P and Romano P 1989 Appl Phys. Lett. 542041

Bednorz J G and Muller K A 1986 Z. Phys. B64 189

Hazen R M 1988 Phys. Rev. Lett. 601174 